\title{
Personal data acquisition IOT gateway
}

\author{
Daniel Vamos ${ }^{(1)}$, Stefan Oniga(1), (2), Anca Alexan (2) \\ (1) Intelligent Embedded Systems Research Laboratory, Department of IT Systems and Networks, Faculty of Informatics, \\ University of Debrecen, Hungary \\ (2) Intelligent Embedded Systems Research Laboratory, Electric, Electronic and Computer Engineering Department, \\ Technical University of Cluj-Napoca, North University Center of Baia Mare, Romania \\ anca.alexan@cunbm.utcluj.ro
}

\begin{abstract}
Personal activity tracker are nowadays part of our lives. They silently monitor our movements and can provide valuable information and even important alerts. But usually the user's data is stored only on the activity tracker device and the processing done is limited by this modest processing power device. Thus it is very important that the user's data can be stored and processed in the cloud, making the activity tracker an IOT node. This paper proposes a simple IOT gateway solution for a custom user monitoring device.
\end{abstract}

Keywords-IOT gateway, activity tracker, CC2650 SimpleLink, personal tracker

\section{INTRODUCTION}

Human activity data gathering is an important research area that has multiple applications in IOT, smart homes and healthcare [1]. This process of gathering, or logging, real-time data has become a normal activity recently due to ubiquitous computing and human activity recognition systems [2].

There are multiple types of method for gathering movement data for a given user, but many of them rely on a complicated and expensive infrastructure, one example is recognizing activity using surveillance cameras [3].

Other attempts to gather activity data are the use of multiple small sensors that are scattered in the user's home. A kind of "tape on and forget" [4] sensors. This means that the sensors are not worn by the user but the environment is able to sense the users' activity. This presents a great drawback as the activity recognition process works only in the environment that is sensor enabled. [5]

Although these complex methods can yield good results, an alternative would be to use accelerometers that are worn by the user [6]. This method allows for recording of precise data in any environment and enables real-time activity detection in any location.

Our approach is to develop a system using two multisensor modules. Since these sensor modules have a wide range of sensors, including accelerometer, gyroscope and magnetometer, we ca correlate and improve the quality of the data that reaches processing.

Our objective is to implement a system capable of handling a decent number of sensors, for a couple of persons, and record data from these sensors in real time. The data should be stored locally but also synchronized with a remote server to allow multiple remote (online or offline) services to process the same data, thus creating a modular and extensible system.

\section{RELATED WORK}

For data acquisition system others have used multiple accelerometer sensors and even combinations of sensors [7] for activity recognition systems. These systems are also used for monitoring human activity. The number of uses sensors, for example accelerometers, differ from one implementation to another, starting from a single sensor and increasing the used sensor number [8].

- [9] uses a single accelerometer to gather and eight activities: brushing teeth, sit-ups, stairs-up, stairs-down, standing, running, vacuuming and walking.

- $\quad$ Three accelerometer sensors were used by [10] to detect recognize lower body movements.

- Five accelerometer sensor were used by [11] in order to increase the system accuracy, being able to recognize 20 types of user activities.

Smartphones are also used in analyzing acceleration data as there are multiple advantages in using them as monitoring devices [12]. Smartphones are carried by the uses almost permanently now-a-days and its single unit approach makes it easy to carry and handle. It has a powerful processing unit capable of not only gathering data but also processing it [13]. Since it also has network capabilities, allowing the phone to be connected to the internet permanently, it can upload the data into the cloud and even send alerts. [14] has used Android based phones worn in the pocket for data acquisition. They analyzed six activities but due to the single sensor system constraint, some activities could not be differentiated.

\section{PROPOSED SYSTEM}

The proposed system consists of the sensor modules, IOT gateway and cloud services as shown in figure 1 .

The system collects movement data using the sensor modules, more precisely accelerometer, gyroscope and magnetometer data. Two multi sensor modules are used, one placed on the user's wrist and the other one placed on the user's leg. The sensors then sends the collected data via Bluetooth to the IOT gateway.

The IOT gateway is represented by an android application that runs on a mobile phone and relays the collected data to a cloud.

In the cloud the data is stored into a relational database making the entire system extensible. 


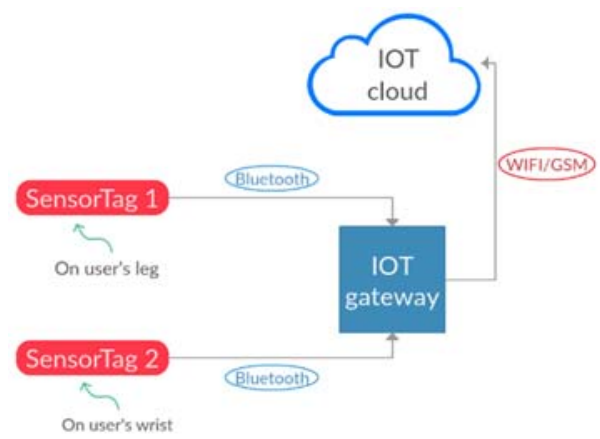

Fig. 1. Proposed system

\section{A. The sensor modules}

The sensor modules used are Texas Instruments CC2650 SimpleLink (figure 2). This module was chosen because it's low power architecture and due to its multiple sensor types [9]:

- Temperature

- Humidity

- Pressure

- Magnetometer

- Gyroscope

- Accelerometer

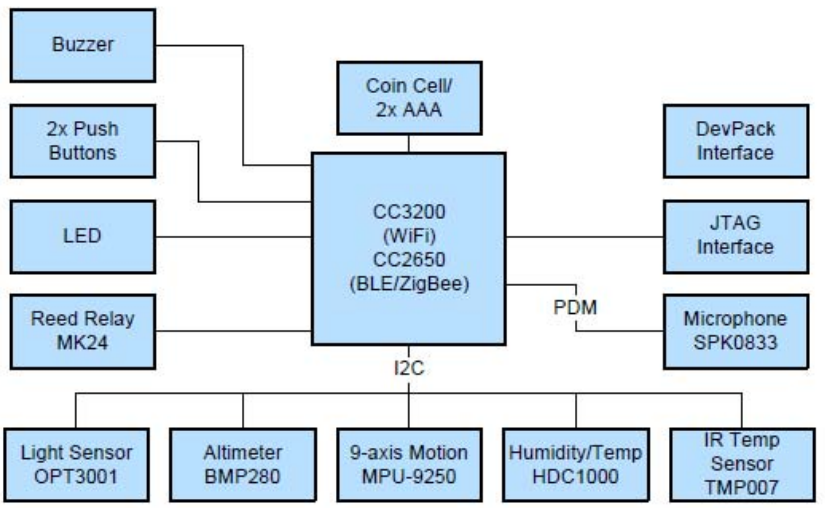

Fig. 2. CC2650 SimpleLink diagram [15]

Since by increasing the number or sensor we can also increase the analysis accuracy, two identical sensor modules were used to obtain movement data. The sensors were placed on the user's wrist and on the user leg above the knee. This placement was chosen to increase the activity recognition rate.

In order to reprogram the Sensor Tag firmware, a SensorTag DevPack board is required. It enables the connection of the Sensor tag module to a PC and allows debugging and updating of the sensor's firmware. Figure 3 shows the SensorTag DevPack board.

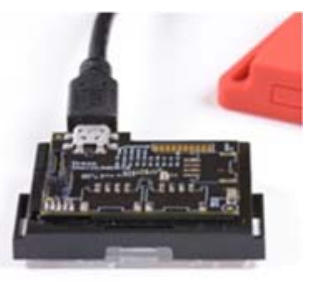

Fig. 3. SensorTag DevPack board
In order to develop software applications for the SensorTag module, two options are available:

- Code Composer Studio

- CCS Cloud

Code Composer Studio represents the offline version of the development tools and features a powerful IDE and debugging capabilities. It's the classical method for programming Texas Instruments devices.

CCS Cloud represents the online version of the IDE and allows development from any web browser. It's portable and simple architecture allows for fast changes and on the fly firmware updates.

The sensor tag firmware was updated using Code Composer Studio IDE in order to increase the number of data sampling from 20 to 50 samples per second. Currently the system uses only acceleration, gyroscope and magnetometer data as this data will be primarily used for activity detection. The rest of the sensor data can be obtained if the IOT gateway application is updated to handle it as the sensorTag modules are currently capable of sending this additional data.

\section{B. The IOT gateway}

The IOT chosen gateway is hardware represented by a smartphone due to its low cost and decent processing power. Also a smart phone is almost always in the proximity of the user making it perfect to also have a gateway role. Also it is WIFI and GSM enabled, being able to upload data into the cloud permanently or at preset intervals.

In the current implementation, a Samsung Note 3 smartphone was used. This phone is Bluetooth enabled, supporting Bluetooth version 4 and runs on an Android 4.3 operating system.

The IOT gateway connects to the sensor modules via Bluetooth and receives sensor data. It then stores the data locally and upload it via internet to the cloud.

An Android gateway software application (figure 4) was implement named "MotionMonitor". It was developed in Android Studio environment.

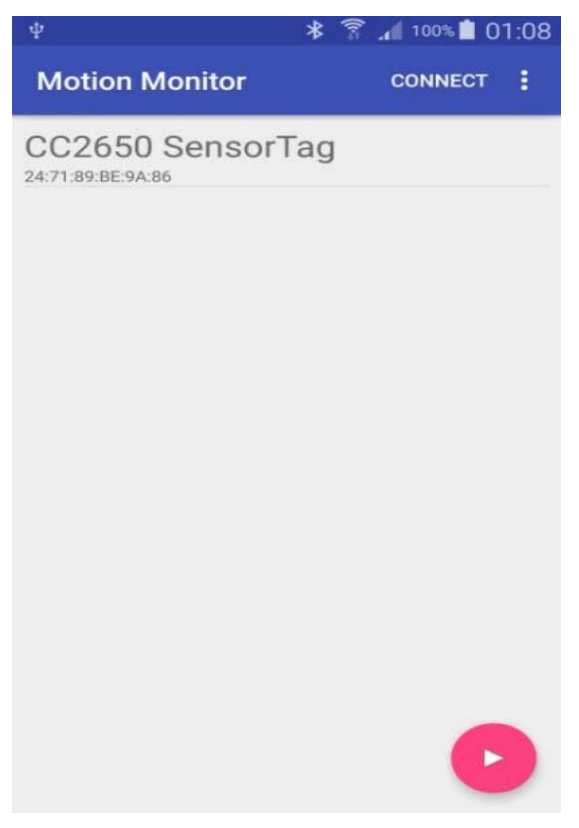

Fig. 4 Android gateway software application 
The "MotionMonitor" software has two main activities: DiscoverActivity and MotionActivity.

The "DiscoverActivity" activity detects the available sensorTag devices via Bluetooth and list them. It allows the user to connect to one or many sensor tag modules. The name of the sensorTag unit is displayed upon detection and in order to differentiate between same name sensors, the sensors mac address is also shown under the name. Since the sensorTag sensor firmware updates are small, our application is able to work with any sensorTag module, even if the firmware is stock.

The "MotionActivity" (figure 5) activity allows viewing and recording sensor data. Three graphs are available on this activity for displaying real time acceleration, gyroscope and magnetometer data. Also the recording button on the right bottom part of the screen displays the recording status.

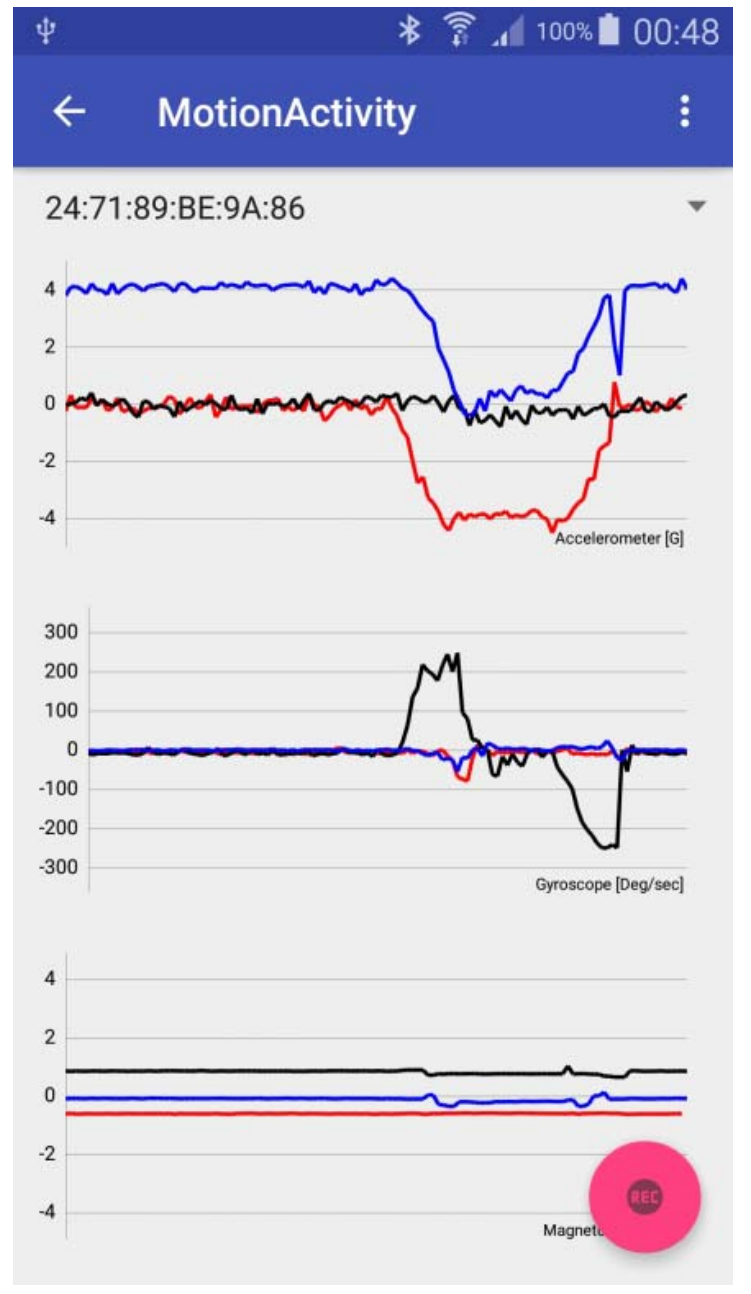

Fig. 5. Activity graphs

Data recording can be started for each sensorTag item and allows for background data recording also. In this way the device is able to perform other tasks in foreground.

The recorded data is also stored locally, in comma separated value files, one file for each sensorTag. This allows for greater flexibility in importing data and also in debugging. The name of the file is composed of the date and the mac address of the sensorTag module, eg. "2018-0630_005015.147_247189BE9A86.csv". The name of the file can be changed in the "Motion record Parameter" screen, as shown in figure 6 , if the user desires to use a different file name. Also, in this screen the record duration in minutes can also be set, the default duration being set to 1 minute. This feature allows the user to set the amount of time the recording process will run, being useful if the process runs in the background and a specific recording period is desired.

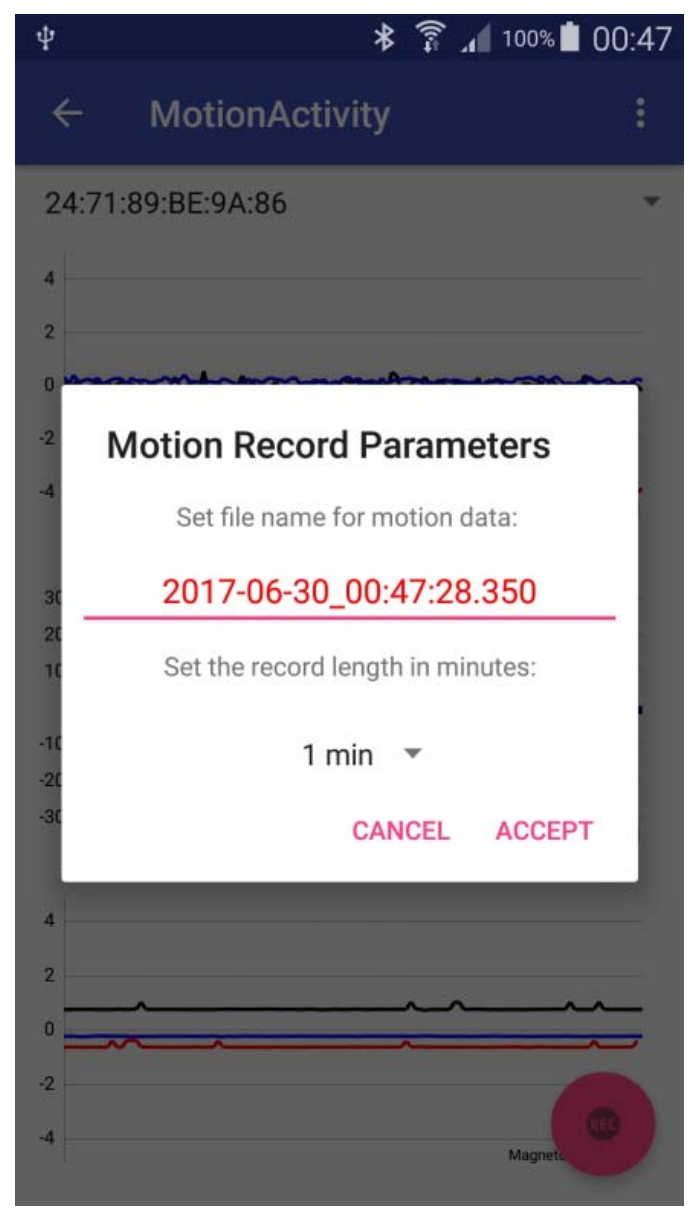

Fig. 6. Setting the recording parameters

The data files stored locally contain for each line

- mac address of the sensorTag

- date and time

- data (three axes)
○ accelerometer
- gyroscope
○ magnetometer

\begin{tabular}{|c|c|c|c|}
\hline Identification data & DATE & TIME & MAC \\
\hline \multirow{3}{*}{ Movement data } & ACC.X & ACC.Y & ACC.Z \\
\cline { 2 - 4 } & GYRO.X & GYRO.Y & GYRO.Z \\
\cline { 2 - 4 } & MAGN.X & MAGN.Y & MANG.Z \\
\hline
\end{tabular}

TABLE I. DATA (THREE AXES)

The same data that is stored in these text files is also up updated to the cloud.

Also the user can specify the interval of recording and also the activity type, for example walking, jogging. 


\section{The cloud}

The cloud portion of the system plays an important role as it creates a common place for data storage.

The central database serves as the common storage place for the activity data. The database used is a relational one, MySql.

The database has multiple tables:

- Subjects table

$\circ$ A table containing the users who are monitored

- Activities table

○ A table containing the activities being monitored

- Sensortags table

○ A table containing the sensorTags used, including identifier the MAC address

- SensorTag_To_Measurements table

○ A linking table between the sensorTags and the data recorded.

- SensorData table

○ A table containg the sensor data, including accelerometer, gyroscope and magnetometer

Since all the movement data are placed in one database, data can be queried easily. We can obtain all historic measurements for a selected activity per user. Also we can easily determine what sensorTags were worn by the user during the recording process. One of the most useful comparison is for the same activity but different users, allowing to spot differences for roughly the same movements.

This central point for data allows for future developed services to retrieve data, as it becomes available, and to process it. Since multiple user data is stored in the same location, for security reasons, the end user will not have access to this location, to prevent accessing data that does not belong to him. Services that will use this database will only return results and no raw data, this way we ensure that the user's data is kept safe.

\section{CONCLUSIONS}

This paper presents the implementation of a complete data acquisition system that is extendable and is IOT orientated. In the center of the system lies the IOT gateway, that is implemented under the form of an Android application. This gateway allows the system to be online, to upload data to the cloud while maintaining a practically zero physical footprint as the phone is in most cases permanently present on the user or in his proximity. The implemented system acquires data from two multi-sensor sensorTag nodes, stores it locally on the IOT gateway and sends it to the cloud. It is extensible as multiple sensors can be added on the fly and supports any stock firmware sensorTag Bluetooth sensor. Also storing data into the cloud opens the system to data manipulation and analysis services integration.

\section{REFERENCES}

[1] A. M. Khan, Y.-K. Lee, S. Y. Lee and T.-S. Kim, "A triaxial accelerometer-based physical-activity recognition via augmented-signal features and a hierarchical recognizer, Information Technology in Biomedicine," IEEE Transactions, 2010.
[2] Gurrin C., Smeaton and Doherty, "LifeLogging: Personal Big Data," Foundations and Trends ${ }^{\circledR}$ in Information Retrieval 8, p. 1-125, 2014.

[3] R. Poppe, "A survey on vision-based human action recognition," Image and vision computing, 2010.

[4] E. Tapia , S. Intille and . K. Larson, "Activity Recognition in the Home Using Simple and Ubiquitous Sensors. In: Ferscha A., Mattern F. (eds) Pervasive Computing. Pervasive 2004.," Lecture Notes in Computer Science, vol. vol 3001. Springer, 2004.

[5] C. Eduardo, . S.-R. Jose Antonio and C.-G. Jose Manuel , "Analysis of a Smartphone-Based Architecture with Multiple Mobility Sensors for Fall Detection," PLoS ONE 11(12): e0168069, 2016.

[6] P. Casale , P. Casale , P. Oriol ,. P. Radeva and O. Petia, "Human activity recognition from accelerometer data using a wearable device, Pattern," Recognition and Image Analysis, 2011.

[7] J. Lester, T. Choudhury and G. Borriello, "A practical approach to recognizing physical activities," Pervasive Computing, 2006.

[8] M. Praveen Kumar, S. Santhoshkumar, T. Gowdhaman and S. Syed Shajahaan, "A SURVEY ON IoT PERFORMANCES IN BIG DATA," International Journal of Computer Science and Mobile Computing ISSN 2320-088X, 2017.

[9] R. N. Littman Michael, N. Dandekar and P. Mysore, "Activity recognition from accelerometer data," $A A A I$, 2005.

[10] K. Narayanan, D. Colbry, C. Juillard and S. Panchanathan, "Real time human activity recognition using tri-axial accelerometers," Sensors, signals and information processing workshop, 2008.

[11] Stephen, Bao Ling and Intille, Pervasive computing. Activity recognition from user-annotated acceleration data, 2004.

[12] Z. Zishan Zahidul Islam, S. Syed Mahir Tazwar, Z. Zahidul Islam, S. Seiichi Serikawa and A. Atiqur Rahman , "Automatic Fall Detection System of Unsupervised Elderly People," Proceedings of the 5th IIAE International Conference on Intelligent Systems and Image Processing 2017, 2017.

[13] S.-R. José Antonio, C. Eduardo and C.-G. José Manuel , "Analysis of a Smartphone-Based Architecture with Multiple Mobility Sensors for Fall Detection with Supervised Learning," Sensors 18(4), 1155, 2018.

[14] K. Jennifer, G. Weiss and S. Moore, "Activity recognition using cell phone accelerometers," $A C M$ SigKDD Explorations Newsletter, 2011.

[15] T. I. Incorporated, Multi-Standard CC2650 SensorTag Design Guide, Texas Instruments Incorporated, 2015. 